\title{
DENDROGRAMA DE MANEJO DA DENSIDADE PARA UMA FLORESTA INEQUIÂNEA DE ARAUCÁRIA
}

\author{
Emanuel Arnoni Costa ${ }^{1 *}$, César Augusto Guimarães Finger ${ }^{2}$, Frederico Dimas Freig ${ }^{2}$, \\ André Felipe Hess ${ }^{1}$, Gabriel Paes Marangon ${ }^{3}$ \\ 1*Universidade do Estado de Santa Catarina, Lages, Santa Catarina, Brasil - emanuelarnonicost@hotmail.com; hessandre@yahoo.com.br \\ ${ }^{2}$ Universidade Federal de Santa Maria, Santa Maria, Rio Grande do Sul, Brasil - cesarfinger.ufsm@gmail.com; dimasfleig@uol.com.br \\ ${ }^{3}$ Universidade Federal de Alagoas, Maceió, Alagoas, Brasil - gabrimarangon@ yahoo.com.br \\ Recebido para publicação: 09/10/2015 - Aceito para publicação: 05/04/2016
}

\begin{abstract}
O objetivo do presente estudo foi desenvolver dendrogramas de manejo da densidade para uma Floresta Inequiânea de Araucária, localizada no município de Lages, SC. Foram amostrados locais com predomínio de árvores de Araucaria angustifolia (Bertol.) Kuntze crescendo sob elevada competição para determinar a área basal por hectare, o número de árvores por hectare, diâmetro de área basal média, volume por hectare, altura dominante e altura média, aplicando o método de densidade pontual proposto por Spurr (1962). O modelo de Reineke (1933) foi ajustado e teve recalculado o valor de intercepto para definir a linha de máxima densidade. O volume médio comercial no ponto amostral foi estimado com o ajuste do modelo de Schumacher e Hall (1933). Todos os modelos foram avaliados quanto a suas estatísticas de ajuste, precisão e análise gráfica dos resíduos. O coeficiente angular do modelo de Reineke (1933) foi de -1,5192, confirmando a Lei da Potência de $-3 / 2$ de autodesbaste. Dendrogramas de manejo desenvolvidos para área basal, volume comercial em função do número de árvores por hectare e diâmetro de área basal média, podem auxiliar nas intervenções florestais, otimizando a produção de acordo com a condição do local e características de crescimento das espécies.

Palavras-chave: Pinheiro-do-Paraná; densidade do povoamento; produção florestal.
\end{abstract}

\begin{abstract}
Density management diagram of Araucaria Uneven Aged Forest. The aim of this study was to develop the density management diagram of Araucaria Uneven Aged Forest, located in Lages city, SC. Plots with predominance of Araucaria angustifolia (Bertol.) Kuntze trees growing under high competition were sampled in order to determine the basal area per hectare, the number of trees per hectare, mean basal area diameter, volume per hectare, dominant height and average height, applying the point density method proposed by Spurr (1962). The Reineke (1933) model was adjusted and had recalculated the intercept value to set the maximum density line. The average trading volume in the sample point was estimated with adjustment of Schumacher and Hall (1933) model modified. All models were evaluated for their adjustment statistics, accuracy and graphical analysis of residuals. Slope coefficient of the Reineke (1933) model was -1.5192, confirming the Law of $-3 / 2$ power of self-thinning. Management dendrograms developed for basal area, commercial volume in function of the number of trees per hectare and average diameter of basal area, can assist in forest operations, optimizing production according to the condition of the site and growth characteristics of the species.

Keywords: Paraná-Pine; stand density; Forest production.
\end{abstract}

\section{INTRODUÇÃO}

Diagramas/Dendrogramas de Manejo da Densidade (DMD) são modelos em nível médio do povoamento que ilustram, graficamente, relações entre produção, densidade e mortalidade, sendo usados para derivar planejamentos de controle da densidade, segundo o objetivo de manejo, e foram desenvolvidos para inúmeras espécies, de interesse comercial em todo o mundo (NEWTON, 1997).

Existem diversos estudos que relatam a confecção e o uso de diagramas de manejo da densidade, destacandose os trabalhos para Abies balsamea (L.) Mill. em florestas de Nova Brunswick, Canadá (PENNER et al., 2006), para Pinus sylvestris L. em florestas do oeste dos Alpes, Itália (VACCHIANO et al., 2008), para Pseudotsuga Menziesii (Mirb.) Franco no norte da Espanha (LÓPEZ-SÁNCHEZ; RODRÍGUEZ-SOALLEIRO, 2009), Pinus radiata D. Don em plantios da Galícia na Espanha norte-ocidental (CASTEDO-DORADO et al., 2009), em povoamentos misto de coníferas em Sierra Nevada, Estados Unidos (LONG; SHAW, 2012), entre outros. 
No Brasil, a confecção de DMD ainda é recente com trabalhos desenvolvidos para povoamentos de Pinus elliottii Engelm (THOMAS, 2009), povoamento de Pinus taeda L. (SCHNEIDER et al., 2009), de Hovenia dulcis Thunberg (SELLE et al., 2010), Araucaria angustifolia (Bertol.) Kuntze (LOUREIRO, 2013), e para Eucalyptus grandis W. Hill. (MARANGON, 2015).

Reineke (1933) propôs o índice de densidade que descreve a relação do tamanho e o número de árvores do povoamento, definindo o valor do coeficiente angular como constante e igual a -1,605, independente do sítio e da espécie. Contudo, estudos posteriores mostraram que o valor do coeficiente angular pode mudar com a espécie, a idade, a classe de copa, entre outros fatores (DEL RÍO et al., 2001; MÁRQUEZ-LINARES; ALVAREZ-ZAGOYA, 1995).

Apesar do DMD ser, frequentemente, desenvolvido para povoamentos puros, seu uso para florestas mistas não pode ser desconsiderado, pois, com os DMD, podem ser obtidas informações para o manejo de árvores individuais, de tal forma que estabeleçam critérios de máxima densidade pontual por hectare, diâmetro máximo alcançado, definição do número máximo de árvores que crescem ao redor da árvore objetivo e que não prejudique o seu desenvolvimento, avaliar a necessidade de plantios de enriquecimento no local com espécies de maior valor comercial, entre outros. Meyer (2011) menciona que, com o conhecimento da máxima densidade, se torna possível manejar a vegetação, evitando a mortalidade e aproveitando a madeira dos cortes seletivos.

Nesse contexto, objetivou-se com o presente estudo, desenvolver dendrogramas de manejo da densidade para uma Floresta Inequiânea de Araucária, no município de Lages, Santa Catarina, como forma de auxiliar e implementar estratégias de manejo florestal e conservação da espécie Araucaria angustifolia.

\section{MATERIAL E MÉTODOS}

\section{Caracterização da área de estudo}

A floresta estudada localiza-se em propriedade particular com 83,5 hectares de Floresta Ombrófila

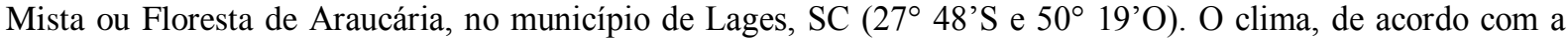
classificação de Köppen, é subtropical úmido, sem estação seca e com verão temperado (Cfb). A Temperatura Anual Média (TAM), é de $15,2^{\circ} \mathrm{C}$, a Precipitação Anual Média (PAM) é de $1684,7 \mathrm{~mm}$ e a altitude média em relação ao nível médio do mar é de 987,0 m (ALVARES et al., 2013). Os solos predominantes na região são Nitossolos Háplicos e Cambissolos Húmicos, desenvolvidos a partir de rochas basálticas (EMPRESA BRASILEIRA DE PESQUISA AGROPECUÁRIA (EMBRAPA), 2006).

Para o desenvolvimento do Plano de Manejo Florestal (PMF) da área do presente estudo, no ano de 2000, utilizou-se o método de área fixa com parcelas retangulares $(10 \mathrm{~m} \times 50 \mathrm{~m})$, totalizando $1,5 \mathrm{ha}$ de amostragem, distribuídas de forma sistemática na área, sendo identificadas 28 espécies, distribuídas em 17 famílias, com Araucaria angustifolia e Ocotea spp. representando maiores dominâncias em relação as demais. (Tabela 1). Para o mesmo local, foi realizada uma proposta de manejo de araucária utilizando o quociente de Liocourt e análise de incremento (HESS et al., 2010), estes autores utilizaram a amostragem por meio do método de área fixa com parcelas $(20 \mathrm{~m}$ x $20 \mathrm{~m})$, totalizando 1 ha de amostragem, distribuídas de forma aleatória na área, a frequência observada foi de 363 araucárias por hectare, abrangendo classes de distribuição diamétrica entre 10,0-70,0 cm, e área basal média de $28,3 \mathrm{~m}^{2} / \mathrm{ha}$.

Tabela 1. Análise fitossociológica horizontal: densidade, dominância, frequência e valor de importância das árvores amostradas na Floresta de Araucária, em Lages, SC.

Table 1. Horizontal phytosociological analysis: density, dominance, frequency and value of importance of trees sampled in Araucaria Forest, in Lages, SC.

\begin{tabular}{|c|c|c|c|c|c|c|}
\hline $\mathbf{N}$ & Nome científico & Família & DR & DoR & FR & VI \\
\hline 1 & Araucaria angustifolia (Bertol.) Kuntze & Araucareaceae & 30,50 & 33,96 & 19,18 & 27,88 \\
\hline 2 & Ocotea spp. & Lauraceae & 27,20 & 29,57 & 13,70 & 23,49 \\
\hline 3 & Sapium glandulosum (L.) Morong & Euphorbiaceae & 5,44 & 4,45 & 8,90 & 6,26 \\
\hline 4 & Piptocarpha sp. & Asteraceae & 4,54 & 3,50 & 8,90 & 5,65 \\
\hline 5 & Lamanonia ternata Vell. & Cunoniaceae & 5,75 & 5,65 & 2,74 & 4,71 \\
\hline 6 & Lithraea brasiliensis Marchand & Anacardiaceae & 2,89 & 2,73 & 6,85 & 4,16 \\
\hline 7 & Styrax leprosus Hook. \& Arn. & Styracaceae & 3,68 & 3,52 & 2,74 & 3,32 \\
\hline 8 & Myrsine umbellata Mart. & Primulaceae & 2,90 & 2,24 & 3,42 & 2,86 \\
\hline 9 & Ilex brevicuspis Reissek & Aquifoliaceae & 1,38 & 1,19 & 4,79 & 2,46 \\
\hline 10 & Myrceugenia euosma (O.Berg) D. Legrand & Myrtaceae & 1,53 & 1,33 & 3,42 & 2,10 \\
\hline 11 & Mimosa scabrella Benth. & Fabaceae & 2,42 & 1,79 & 2,05 & 2,09 \\
\hline 12 & Gymnanthes klotzschiana Müll.Arg. & Euphorbiaceae & 2,40 & 2,03 & 1,37 & 1,93 \\
\hline 13 & Handroanthus heptaphyllus (Mart.) Mattos & Bignoniaceae & 1,39 & 1,42 & 2,74 & 1,85 \\
\hline 14 & NI1 & & 1,76 & 1,25 & 2,05 & 1,69 \\
\hline
\end{tabular}




\begin{tabular}{llccccc}
\hline 15 & Zanthoxylum sp. & Rutaceae & 1,02 & 0,96 & 2,74 & 1,57 \\
16 & Drimys brasiliensis Miers & Winteraceae & 0,76 & 0,54 & 2,05 & 1,12 \\
17 & Ilex paraguariensis A. St.-Hil. & Aquifoliaceae & 0,64 & 0,43 & 2,05 & 1,04 \\
18 & Casearia decandra Jacq. & Salicaceae & 0,77 & 0,64 & 1,37 & 0,93 \\
19 & Gochnatia polymorpha (Less.) Cabr. & Asteraceae & 0,38 & 0,33 & 1,37 & 0,69 \\
20 & Schinus terebinthifolius Raddi & Anacardiaceae & 0,25 & 0,45 & 1,37 & 0,69 \\
21 & Psidium guajava L. & Myrtaceae & 0,38 & 0,26 & 1,37 & 0,67 \\
22 & Dalbergia frutescens (Vell.) Britton & Fabaceae & 0,64 & 0,54 & 0,68 & 0,62 \\
23 & Cupania vernalis Cambess. & Sapindaceae & 0,50 & 0,36 & 0,68 & 0,52 \\
24 & Cedrela fissilis Vell. & Meliaceae & 0,26 & 0,24 & 0,68 & 0,39 \\
25 & Eugenia pyriformis Cambess. & Myrtaceae & 0,26 & 0,19 & 0,68 & 0,38 \\
26 & Blepharocalyx salicifolius (Kunth) O.Berg & Myrtaceae & 0,12 & 0,20 & 0,68 & 0,34 \\
27 & NI2 & & 0,12 & 0,13 & 0,68 & 0,31 \\
28 & Baccharis dracunculifolia DC. & Asteraceae & 0,12 & 0,08 & 0,68 & 0,30 \\
\hline & & Total & 100,0 & 100,0 & 100,0 & 100,0 \\
\hline
\end{tabular}

em que: $\mathrm{N}=$ número de espécies; $\mathrm{DR}=$ densidade relativa, em \%; DoR = dominância relativa, em \%; FR = frequência relativa, em \%; VI = valor de importância, em \%; NI = não identificada.

\section{Construção dos dendrogramas de manejo da densidade}

Para a construção dos dendrogramas de manejo da densidade foram utilizadas 82 unidades amostrais, distribuídas no interior da floresta natural no ano de 2014, aplicando o método de Densidade Pontual proposto por Spurr (1962). Desta forma, foi possível quantificar a área basal por hectare (G/ha), número de árvores por hectare (N/ha), diâmetro de área basal média $(\mathrm{dg})$, volume por hectare $(\mathrm{V} / \mathrm{ha})$ e altura dominante $\left(\mathrm{h}_{\mathrm{dom}}\right)$. Durante esta amostragem, as espécies folhosas não foram identificadas taxonomicamente, apenas houve a distinção entre árvores de araucária e folhosas, uma vez que, nesse local havia uma grande dominância desta espécie conforme observado no Plano de Manejo Florestal (PMF) realizado ano de 2000 (Tabela 1), o que também foi constatado no levantamento do presente estudo realizado no ano de 2014.

O critério de inclusão das árvores na parcela foi o mesmo descrito por Bitterlich, que se baseia em um ângulo crítico que define um Fator de Área Basal (FAB), para calcular a distância da árvore em análise em relação às suas respectivas concorrentes, em função do DAP:

$$
\mathrm{L}=\frac{0,5 \text { DAP }}{\sqrt{\mathrm{FAB}}}
$$

em que: $\mathrm{L}=$ distância radial da árvore objetivo em relação a árvore com suposição de ser concorrente, em m; $\mathrm{DAP}=$ diâmetro à altura do peito, em $\mathrm{cm} ; \mathrm{FAB}=2$ ou 4 , usado na amostragem.

A área basal por hectare (G/ha), segundo o método de Densidade Pontual proposto por Spurr (1962), é calculada por:

$$
\mathrm{G} / \mathrm{ha}=\frac{\sum_{\mathrm{j}=1}^{\mathrm{n}}\left\{(\mathrm{j}-0,5)\left[0,25\left(\frac{\mathrm{DAP}_{\mathrm{j}}}{\mathrm{DH}_{\mathrm{j}}}\right)^{2}\right]\right\}}{\mathrm{n}}
$$

em que: $\mathrm{G} / \mathrm{ha}=$ área basal por hectare excluindo a árvore em análise; $\mathrm{DH}_{\mathrm{j}}=$ distância horizontal da árvore análise em relação à árvore com suposição de ser j-ésima concorrente, em m; DAP ${ }_{j}=$ diâmetro à altura do peito da jésima árvore com suposição de ser concorrente, $\mathrm{em} \mathrm{cm} ; \mathrm{n}=$ número total de árvores concorrentes situadas ao redor da árvore em análise; FAB = fator de área basal igual a 2 ou 4 usado na amostragem.

O número de árvores por hectare (N/ha) foi obtido com base no método de Bitterlich, calculado por:

$$
\mathrm{N} / \mathrm{ha}=\mathrm{FAB} \sum_{\mathrm{j}=1}^{\mathrm{n}} \frac{1}{\mathrm{~g}_{\mathrm{j}}}
$$

em que: $\mathrm{N} / \mathrm{ha}=$ número de árvores por hectare; $\mathrm{g}_{\mathrm{j}}=$ área basal da j-ésima árvore medida no ponto amostral, em $\mathrm{m}^{2} ; \mathrm{FAB}=$ fator de área basal igual a 2 ou 4 usado na amostragem.

O diâmetro de área basal média (dg) foi calculado dividindo a área basal de cada ponto amostral pelo número de árvores por hectare, conforme a expressão:

$$
\mathrm{dg}=\sqrt{\left[4 .\left(\frac{\mathrm{G} / \mathrm{ha}}{\mathrm{N} / \mathrm{ha}}\right) / \pi\right]} \cdot 100
$$

em que: $\mathrm{dg}=$ diâmetro de área basal média, em $\mathrm{cm} ; \mathrm{G} / \mathrm{ha}=$ área basal por hectare; $\mathrm{N} / \mathrm{ha}=$ número de árvores por hectare; $\pi=$ constante aproximadamente 3,1416 . 
Para estimar o volume comercial de cada árvore de araucária amostrada, foi ajustado o modelo (5) de Schumacher e Hall (1933). Os dados para o ajuste da equação foram obtidos de cubagem rigorosa de 85 araucárias com o aparelho Criterion RD 1000 e aplicado o método de Hohenadl para as alturas específicas $\left(\mathrm{h}_{\mathrm{i}}\right)$ de $10 \%, 30 \%, 50 \%, 70 \%$ da altura total (h), bem como o diâmetro no ponto de inserção da copa (dic). O diâmetro a $90 \%$ da altura não foi considerado por estar sempre localizado dentro da copa e por existir a presença de galhos que impedia sua determinação dentro da floresta. $\mathrm{O}$ volume comercial das demais espécies folhosas foi calculado de forma direta para cada árvore pela expressão (6).

Araucárias:

$$
\mathrm{vc}=0,000063 \mathrm{DAP} \mathrm{P}^{2,111273} \mathrm{~h}^{0,705521} \quad\left(\mathrm{R}^{2}=0,9805 ; \mathrm{Syx} \%=12,04\right)
$$

Folhosas:

$$
\mathrm{vc}=\frac{\left(\pi \cdot \mathrm{DAP}^{2}\right)}{40000} \cdot \mathrm{h}_{\mathrm{ic}} \cdot \mathrm{f}_{\mathrm{c}}
$$

em que: $\mathrm{vc}=$ volume comercial; $\mathrm{em} \mathrm{m}^{3}$ com casca; $\pi=$ constante aproximadamente 3,$1416 ; \mathrm{DAP}=$ diâmetro à altura do peito, em $\mathrm{cm} ; \mathrm{h}=$ altura total, em $\mathrm{m} ; \mathrm{h}_{\mathrm{ic}}=$ altura até o ponto de inserção da copa, em $\mathrm{m} ; \mathrm{f}_{\mathrm{c}}=$ fator de forma comercial médio $(0,77)$, determinado para calcular os volumes individuais das espécies folhosas em floresta de araucária (BORSOI, 2004).

O volume comercial por hectare $\left(\mathrm{V} / \mathrm{ha}_{\mathrm{c}}\right)$ do ponto amostral foi obtido pela multiplicação do fator de área basal (FAB) usado com o inverso da área basal da j-ésima árvore medida no ponto amostral, esta razão refere-se ao número de árvores por hectare que a árvore representa no ponto amostral, multiplicado pelo seu respectivo volume $\left(\mathrm{v}_{\mathrm{j}}\right)$, conforme a expressão:

$$
V / h a_{c}=F A B \sum_{j=1}^{n} \frac{1}{g_{j}} v_{j}
$$

em que: $\mathrm{V} / \mathrm{ha}_{\mathrm{c}}=$ volume comercial por hectare para araucária e demais espécies folhosas, em $\mathrm{m}^{3}$ com casca; $\mathrm{g}_{\mathrm{j}}=$ área basal da j-ésima árvore medida no ponto amostral, em m²; FAB = fator de área basal igual a 2 ou 4 usado na amostragem; $\mathrm{v}_{\mathrm{j}}=$ volume calculado para cada árvore pertencente ao ponto amostral com as respectivas equações 5 e 6 , em $\mathrm{m}^{3}$, com casca.

Para estimar a altura dominante $\left(\mathrm{h}_{\mathrm{dom}}\right)$, foi calculada a média aritmética das alturas totais das três árvores mais grossas de cada ponto amostral, conforme a expressão:

$$
\mathrm{h}_{\mathrm{dom}}=\sum_{\mathrm{j}=1}^{\mathrm{n}=3} \frac{\mathrm{h}}{\mathrm{n}}
$$

em que: $\mathrm{h}_{\mathrm{dom}}=$ altura dominante, em $\mathrm{m} ; \mathrm{h}=$ altura total, $\mathrm{em} \mathrm{m}$.

A amostragem permitiu descrever a relação entre o número de árvores por hectare (N/ha) em função do diâmetro de área basal média (dg), por meio do modelo de Reineke (1933), na forma linear, conforme a expressão:

$$
\ln (\mathrm{N} / \mathrm{ha})=\beta_{0}+\beta_{1} \ln (\mathrm{dg})+\varepsilon
$$

em que: $\mathrm{N} / \mathrm{ha}=$ número de árvores por hectare; $\mathrm{dg}=$ diâmetro de área basal média, em $\mathrm{cm} ; \beta_{0}$ e $\beta_{1}=$ coeficientes de regressão estimados; $\varepsilon=$ erro residual.

No estabelecimento do DMD, a linha A, que descreve a máxima densidade de ocupação "linha de autodesbaste", foi definida após o ajuste da equação (9) e do recálculo de novos valores de intercepto $\left(\beta_{0}\right)$ até o momento em que não houvesse mais dados acima deste limite. Para isso, foi considerado um diâmetro de área basal (dg) de 25,0 cm. Esta linha de máxima é, frequentemente, usada como indicador da capacidade máxima que o local suporta (SELLE et al., 2010).

A faixa de manejo compreendida entre a linha B “início da mortalidade” com a linha C "máxima produtividade" foi fixada em relação à linha A, considerando valores de 57,5\% para a linha B e 42,5\% para linha C, ficando a faixa de manejo estipulada em uma amplitude de 15\%. Os trabalhos de Schneider et al. (2009), Selle et al. (2010), Schneider et al. (2011) e Loureiro (2013) recomendaram valores entre 55,0\% (Linha B) e $25,0 \%$ (Linha C) em relação à máxima (Linha A) como faixa ideal de manejo. No entanto, esta amplitude de manejo pode sofrer alteração conforme os objetivos estabelecidos no manejo da floresta. 
A linha D que descreve a "ocupação das copas" foi baseada no levantamento do diâmetro de copa (dc), de acordo com a metodologia proposta por Krajicek et al. (1961), que a definiu como uma medida de densidade própria de um povoamento independentemente da idade e da qualidade do sítio.

Devido à amostragem dos dados ser em floresta com predomínio de árvores de araucária, utilizou-se um modelo de regressão do diâmetro de copa (dc) em função do DAP da espécie para descrever a linha D. De acordo com os resultados encontrados por Costa (2015), onde não se encontrou diferenças significantes nesta relação entre árvores de crescimento livres e sob competição em floresta natural de diferentes locais no sul do Brasil, valeu-se da equação (10) como sendo a potencial para a espécie:

$$
\mathrm{dc}_{\text {potencial }}=4,8601+0,2038 \mathrm{DAP}
$$

em que: $\mathrm{dc}_{\text {potencial }}=$ diâmetro de copa potencial, em m; DAP = diâmetro à altura do peito, em $\mathrm{cm}$.

Assim, a linha D que descreve a ocupação das copas foi estabelecida em função da equação (10), conforme a metodologia proposta por Krajicek et al. (1961), sendo calculada da seguinte forma:

$$
\mathrm{N} / \mathrm{ha}=\frac{40000}{\pi\left(\mathrm{dc}_{\text {potencial }}\right)^{2}}
$$

em que: $\mathrm{N} / \mathrm{ha}=$ número de árvores por hectare $; \mathrm{dc}_{\text {potencial }}=$ diâmetro de copa potencial, em $\mathrm{m} ; \pi=$ constante aproximadamente 3,1416 .

\section{Determinação do volume médio comercial no ponto amostral}

O Volume Médio Comercial (VMC) das árvores no ponto amostral foi obtido pela razão entre o volume comercial por hectare $\left(\mathrm{V} / \mathrm{ha}_{\mathrm{c}}\right)$ (Expressão 7) pelo número de árvores por hectare (N/ha) (Expressão 3). Com estes valores calculados em cada ponto amostral, foi possível modificar o modelo de Schumacher e Hall (1933) (Expressão 12) e ajustar por regressão não linear esta relação em função das variáveis diâmetro de área basal média $(\mathrm{dg})$ (Expressão 4), altura dominante $\left(\mathrm{h}_{\mathrm{dom}}\right)$ (Expressão 8) e a média aritmética das altura $\left(\mathrm{h}_{\mathrm{m}}\right)$ das árvores pertencentes ao ponto amostral.

$$
\mathrm{vmc}=\beta_{0} \mathrm{dg}^{\beta_{1}}\left(\frac{\mathrm{hm}}{\mathrm{h}_{\mathrm{dom}}}\right)^{\beta_{2}}+\varepsilon
$$

em que: vmc $=$ volume médio comercial no ponto amostral, $\mathrm{em}^{3}$ com casca $; \mathrm{dg}=$ diâmetro de área basal média no ponto amostral, em $\mathrm{cm} ; \mathrm{hm}=$ altura total média das árvores no ponto amostral, em $\mathrm{m} ; \mathrm{h}_{\mathrm{dom}}=$ altura dominante no ponto amostral, em $\mathrm{m} ; \beta_{0}, \beta_{1}, \beta_{2}=$ coeficientes de regressão estimados; $\varepsilon=$ erro residual.

A relação entre a altura dominante $\left(\mathrm{h}_{\mathrm{dom}}\right)$ e o diâmetro de área basal média $(\mathrm{dg})$ foi descrita com o ajuste do modelo de Michailoff (1943):

$$
\mathrm{h}_{\mathrm{dom}}=1,3+\beta_{0} \exp \left(-\frac{\beta_{1}}{\mathrm{dg}}\right)+\varepsilon
$$

em que: $\mathrm{h}_{\mathrm{dom}}=$ altura dominante no ponto amostral, em $\mathrm{m} ; \mathrm{dg}=$ diâmetro de área basal média no ponto amostral, em cm; $\beta_{0}$ e $\beta_{1}=$ coeficientes de regressão estimados; $\varepsilon=$ erro residual.

A altura média $(\mathrm{hm})$ das árvores de araucária e folhosas pertencentes ao ponto amostral foi descrito com o modelo modificado de Michailoff (1943):

$$
\mathrm{hm}=1,3+\left(\beta_{0}+\beta_{1} \mathrm{~h}_{\mathrm{dom}}\right) \exp \left(-\frac{\beta_{2}}{\mathrm{dg}}\right)+\varepsilon
$$

em que: $\mathrm{hm}=$ altura total média das árvores no ponto amostral, em $\mathrm{m} ; \mathrm{h}_{\mathrm{dom}}=$ altura dominante no ponto amostral, em $\mathrm{m} ; \mathrm{dg}=$ diâmetro de área basal média no ponto amostral, em $\mathrm{cm} ; \beta_{0}, \beta_{1}, \beta_{2}=$ coeficientes de regressão estimados; $\varepsilon=$ erro residual.

\section{Análise dos dados}

Os modelos foram ajustados no Sistema de Análise Estatística (SAS, 2004) e o coeficiente de determinação $\left(\mathrm{R}^{2}\right)$, o erro padrão da estimativa em porcentagem $($ Syx \%) e a análise gráfica dos resíduos em função dos valores estimados, foram usados para verificar a performance das equações. A PROC REG foi usada para a estimativa dos coeficientes de regressão com o Método dos Mínimos Quadrados Ordinários. A PROC MODEL foi usada para a estimativa dos coeficientes de regressão com o Método dos Mínimos Quadrados Ordinários Não Linear, que buscou a solução de equações através do método iterativo de Marquardt. 


\section{RESULTADOS}

\section{Características das árvores amostradas}

A amostragem de 82 pontos amostrais com elevada competição distribuídos no interior da floresta natural, aplicando o método de Densidade Pontual de Spurr, permitiu encontrar em 30 pontos amostrais somente a presença de araucária (Figura 1). Em média, foram medidos 6,99 árvores de araucária em cada ponto amostral, com mínimo de 2,0 e máximo de 16,0. Em apenas 17,0\% dos pontos amostrais, foram encontradas árvores folhosas, em média 1,57 folhosas em cada ponto amostral.

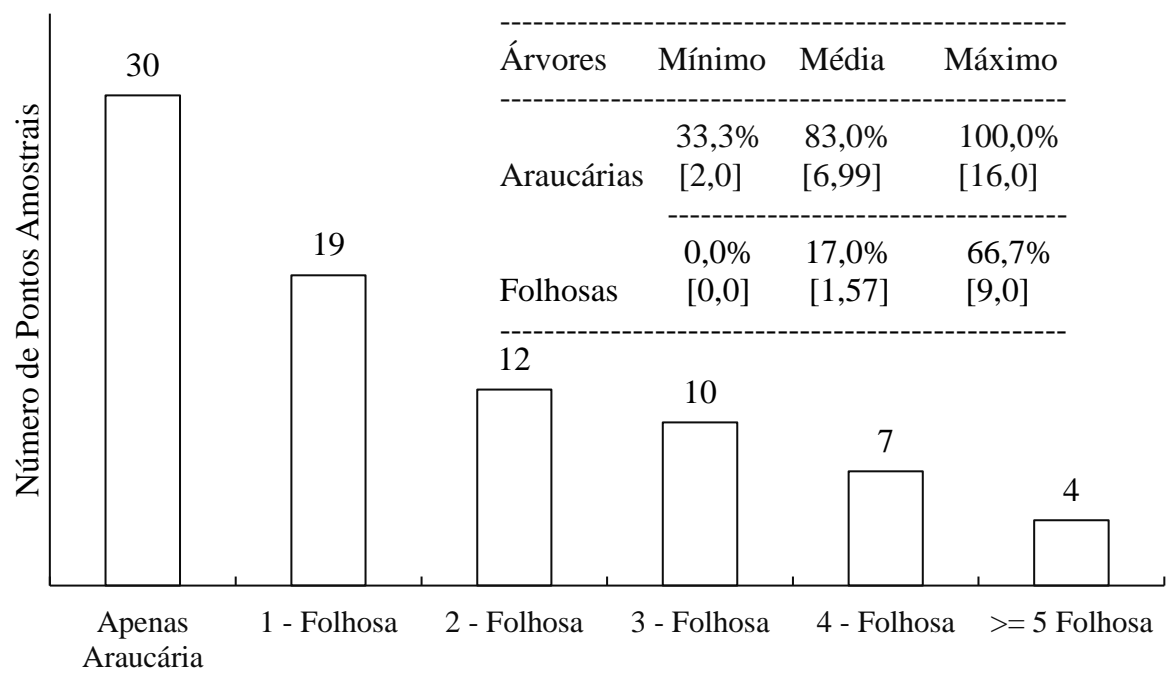

Figura 1. Número de pontos amostrais com apenas a presença de araucária, 1, 2, 3, 4 e $\geq 5$ indivíduos de folhosas em uma Floresta de Araucária, em Lages, SC. [..] = em colchetes o número de árvores de araucária ou folhosas medidas nos pontos amostrais.

Figure 1. Number of sample points with only the presence of araucaria, 1, 2, 3, 4 and $\geq 5$ hardwood individuals in Araucaria Forest, in Lages, SC. [..] = In brackets the number of araucaria trees or hardwood measures in the sample points.

O resumo estatístico das características das árvores em cada ponto amostral (Tabela 2) mostrou a G/ha média de $30,6 \mathrm{~m}^{2}$.ha $\mathrm{ha}^{-1}$ com máximo atingindo $62,5 \mathrm{~m}^{2} \cdot \mathrm{ha}^{-1}$, mostrando um povoamento superestocado. Os maiores valores de $\mathrm{hm} \mathrm{e} \mathrm{h}_{\text {dom }}$ foram 20,2 e 22,2 m, respectivamente, e o valor mínimo do V/ha foi $66,8 \mathrm{~m}^{3} \cdot \mathrm{ha}^{-1}$, com média de $275,9 \mathrm{~m}^{3} \cdot \mathrm{ha}^{-1}$ e máximo de $610,9 \mathrm{~m}^{3} \cdot \mathrm{ha}^{-1}$.

Tabela 2. Resumo estatístico das características das árvores amostradas nos pontos amostrais em uma Floresta de Araucária, em Lages, SC.

Table 2. Statistical summary of e characteristics of trees sampled in the sampling points in a Araucaria Forest, in Lages, SC.

\begin{tabular}{lcccc}
\hline Variável & Mínimo & Média & Máximo & CV\% \\
\hline $\mathrm{dg}$ & 14,0 & 29,4 & 53,4 & 24,0 \\
$\mathrm{hm}$ & 10,3 & 15,4 & 20,2 & 17,0 \\
$\mathrm{~h}_{\mathrm{dom}}$ & 10,7 & 17,5 & 22,2 & 17,5 \\
$\mathrm{G} / \mathrm{ha}$ & 5,6 & 30,6 & 62,5 & 48,0 \\
$\mathrm{~N} / \mathrm{ha}$ & 90,2 & 469,8 & 904,1 & 44,7 \\
$\mathrm{~V} / \mathrm{ha}$ & 66,8 & 275,9 & 610,9 & 48,1 \\
$\mathrm{vmc}$ & 0,1508 & 0,6678 & 1,9650 & 53,4 \\
\hline
\end{tabular}

em que: $\mathrm{dg}=$ diâmetro de área basal média, em $\mathrm{cm}$; $\mathrm{hm}=$ altura total média, em $\mathrm{m} ; \mathrm{h}_{\mathrm{dom}}=$ altura dominante, em $\mathrm{m}$; $\mathrm{G} / \mathrm{ha}=$ área basal por hectare; $\mathrm{N} / \mathrm{ha}=$ número de árvores por hectare; $\mathrm{V} / \mathrm{ha}=$ volume por hectare, $\mathrm{em}^{3}$ com casca; vmc $=$ volume médio comercial, em $\mathrm{m}^{3} \mathrm{com}$ casca; $\mathrm{CV} \%=$ coeficiente de variação.

\section{Determinação da Linha A}

Com o ajuste do modelo de Reineke (1933) na forma linear (expressão 9), foi possível descrever a tendência média do $\ln (\mathrm{N} / \mathrm{ha})$ em função do $\ln (\mathrm{dg})$ (Figura 2), apresentando regressão significante $($ Fvalor $=$ 
$35,1 ; \mathrm{p}<0,0001)$, ajuste baixo $\left(\mathrm{R}^{2}=0,3473\right)$ e alta precisão $($ Syx $\%=6,72)$. O valor do coeficiente angular foi de $\left(\beta_{1}=1,5192\right)$, confirmando a lei da "Potência". A Linha A - "Autodesbaste" foi definida alterando o valor de intercepto de $\left(\beta_{0}=11,2507\right)$ para $\left(\beta_{0}=11,9507\right)$, estabelecido a partir de um dg padrão de $25,0 \mathrm{~cm}$.

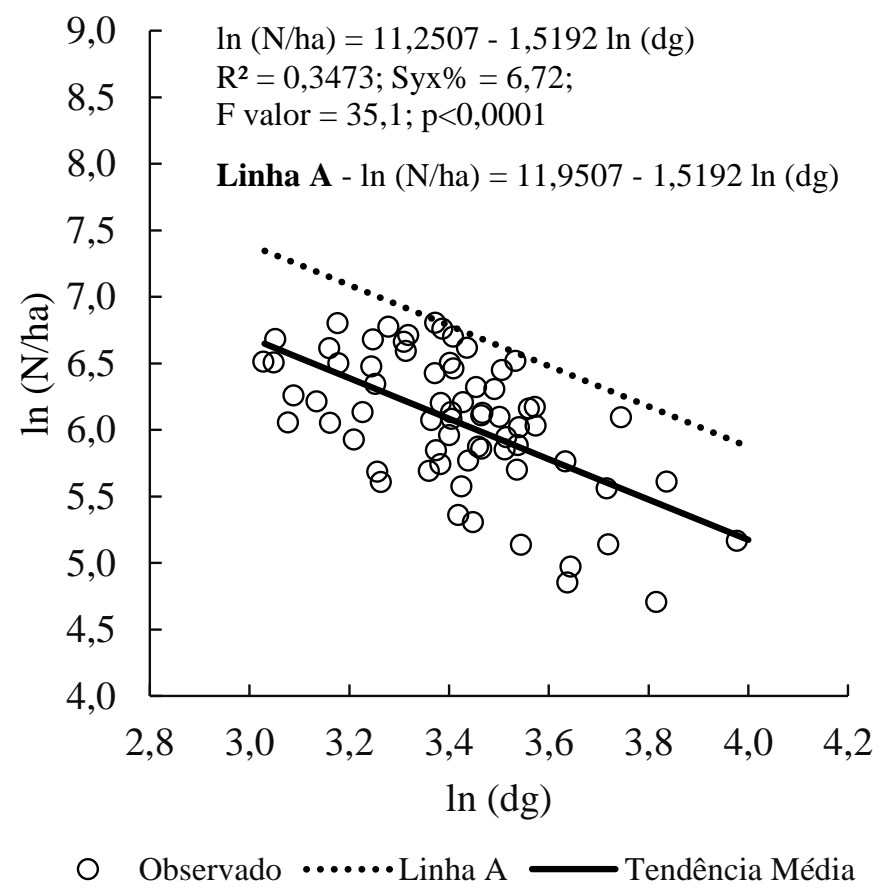

Figura 2. Ajuste do modelo de Reineke (1933) para descrever o ln (N/ha) em função do ln (dg). A Linha A "Autodesbaste" foi definida alterando o valor de intercepto $\left(\beta_{0}\right)$ do modelo da Tendência média para uma Floresta de Araucária, em Lages, SC.

Figure 2. Adjusting Reineke (1933) model to describe the ln (N/ha) versus ln (dg). Line A - "self-thinning" was defined by changing the intercept value $\left(\beta_{0}\right)$ of average model tendency for Araucaria Forest, in Lages, SC.

\section{Ajuste de modelos para vmc, $\mathbf{h}_{\text {dom }}$ e hm.}

Os três modelos ajustados para vmc, $h_{\text {dom }}$ e $h m$ alcançaram significância $(\alpha=5 \%)$ para todos os coeficientes de regressão estimados (Tabela 3). Os modelos de vmc e hm apresentaram os melhores valores quanto aos critérios de ajuste $\left(\mathrm{R}^{2}\right)$ e precisão (Syx \%).

A análise gráfica dos resíduos em função dos valores estimados (Figura 3) para os três modelos ajustados mostrou distribuição regular dos erros sem a presença de tendências sistemáticas, que superestimassem ou subestimassem valores, confirmando a seleção dos modelos da tabela 3.

Tabela 3. Coeficientes de regressão estimados e estatísticas de ajuste e precisão para o vmc, $\mathrm{h}_{\mathrm{dom}}$ e hm para uma Floresta de Araucária, em Lages, SC.

Table 3. Estimated regression coefficients and fit statistics and accuracy for vmc, hdom and hm for Araucaria Forest, in Lages, SC.

\begin{tabular}{lcccccc}
\hline Equação & Variável & $\boldsymbol{\beta}_{\mathbf{0}}$ & $\boldsymbol{\beta}_{\mathbf{1}}$ & $\boldsymbol{\beta}_{\mathbf{2}}$ & $\mathbf{R}^{\mathbf{2}}$ & Syx \% \\
\hline \multirow{2}{*}{12} & $\mathrm{vmc}$ & 0,00188 & 1,76857 & 1,18245 & 0,7983 & 24,62 \\
& & $(0,00075)$ & $(0,1097)$ & $(0,3457)$ & & \multirow{2}{*}{$0,38,83$} \\
\multirow{2}{*}{13} & 25,79118 & 12,98256 & - & & \\
\multirow{2}{*}{14} & $\mathrm{~h}_{\mathrm{dom}}$ & $(1,8008)$ & $(1,9897)$ & - & & \multirow{2}{*}{7,45} \\
& \multirow{2}{*}{$\mathrm{hm}$} & 3,15542 & 0,76060 & 4,57761 & \multirow{2}{*}{0,8117} & $(1,3631)$ \\
\hline
\end{tabular}

em que: $v m c=$ volume médio comercial, em $\mathrm{m}^{3}$ com casca; $\mathrm{h}_{\mathrm{dom}}=$ altura dominante, em $\mathrm{m} ; \mathrm{hm}=$ altura total média, em m; $\beta_{0}, \beta_{1}, \beta_{2}=$ coeficientes de regressão; (..) valor em parênteses indica o erro padrão dos coeficientes estimados; $\mathrm{R}^{2}=$ coeficiente de determinação; Syx $\%=$ erro padrão da estimativa em porcentagem. 

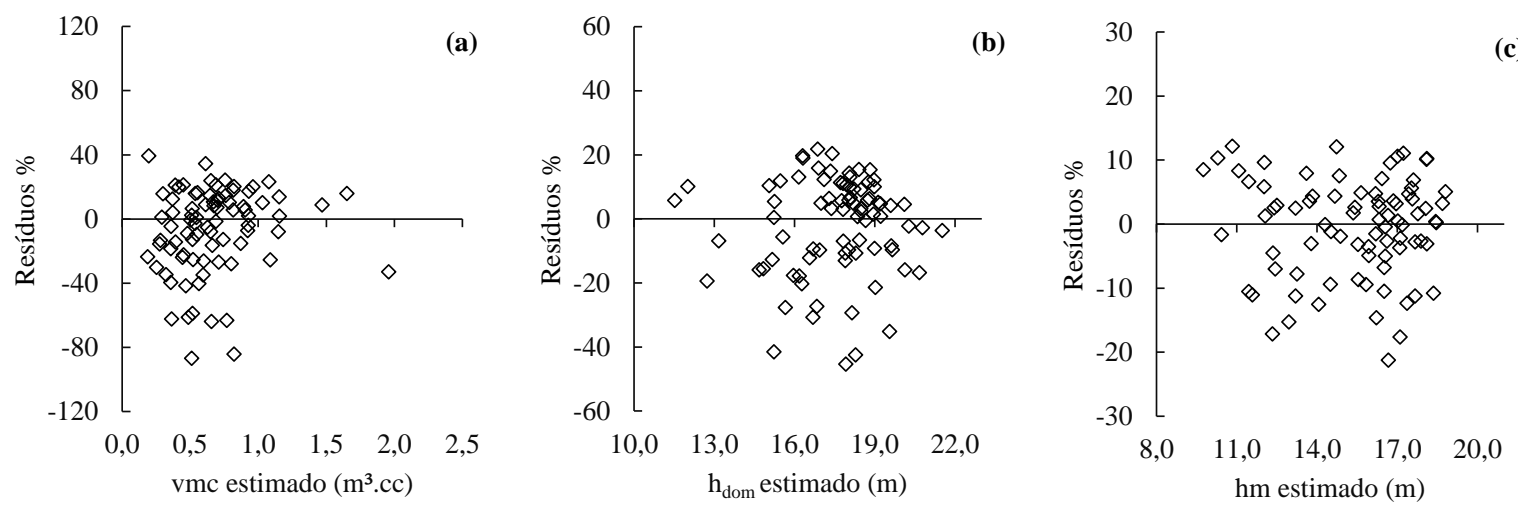

(c)

Figura 3. Dispersão dos resíduos $\%$ em função dos valores estimados. (a) - vmc $=$ volume médio comercial, em $\mathrm{m}^{3} . \mathrm{cc} ;$ (b) $-\mathrm{h}_{\mathrm{dom}}=$ altura dominante, em $\mathrm{m}$; (c) $-\mathrm{hm}=$ altura média, em $\mathrm{m}$; para uma Floresta de Araucária, em Lages, SC.

Figure 3. Dispersion of residuals $\%$ in function of estimate values (a) $-v m c=$ average comercial volume in $\mathrm{m}^{3} . \mathrm{cc}$; (b) - hdom = dominant height, in meters; (c) - $\mathrm{hm}=$ average height, in meters; for Araucaria Forest, in Lages, SC.

\section{Dendrogramas de Manejo da Densidade (DMD)}

O dendrograma de manejo desenvolvido para a área basal por hectare, a partir de interação das variáveis $\mathrm{N} / \mathrm{ha}$ e dg, ficou definido na figura 4 . A faixa de manejo foi estipulada em uma amplitude de $15 \%$ entre as Linhas diretrizes B e C, preestabelecidas entre valores de 57,5\% e 42,5\% em relação a Linha A (Expressão 9).

De forma similar ao DMD anterior, foi desenvolvido o DMD para $\mathrm{V} / \mathrm{ha}$, dado pela interação das variáveis N/ha e dg (Figura 5). Com a expressão (12), foi estimado o volume médio comercial (vmc) de cada árvore pertencente ao ponto amostral e com as expressões (13) e (14), as alturas hdom e hm.

\section{Simulação de desbastes com o Diagrama de Manejo da Densidade}

A simulação das atividades desenvolvidas no povoamento foram apresentadas com base nas equações ajustadas no presente estudo. De acordo com as características das árvores amostradas nos pontos amostrais (Tabela 2), foram simulados desbastes (Tabela 4), considerando os objetivos de regular a produção da floresta, almejando o máximo aproveitamento da produção por meio do planejamento das atividades a serem implementadas.

A título de exemplo, encontrou-se, no ponto amostral, uma G/ha de $40,0 \mathrm{~m}^{2} \cdot \mathrm{ha}^{-1}$, dg de $30 \mathrm{~cm}$ e N/ha de 550,0 aplicando o método de Densidade Pontual de Spurr. Com base nesses valores, foram definidos desbastes moderados, fazendo-se uma regulação de $35,0 \%$ da G/ha, atualmente, encontrada, equivalente à redução de 14,0 $\mathrm{m}^{2}$. ha ${ }^{-1}$ e N/ha de 175,0 (Tabela 4).

Tabela 4. Simulação da execução de desbastes em Floresta de Araucária, em Lages, SC.

Table 4. Simulation thinning execution in Araucaria Forest in Lages, SC.

\begin{tabular}{|c|c|c|c|c|c|c|c|c|c|}
\hline \multirow{2}{*}{ dg (cm) } & \multirow{2}{*}{ Anos } & \multirow{2}{*}{$\mathbf{h}_{\text {dom }}$} & \multirow{2}{*}{ hm } & \multicolumn{2}{|c|}{ Remanescente } & \multicolumn{4}{|c|}{ Desbaste } \\
\hline & & & & N/ha & G (m²/ha) & N/ha & G (m²/ha) & $\%$ N/ha & $\%$ G (m²/ha) \\
\hline 30,0 & - & 18,0 & 15,8 & 550,0 & 40,0 & & & & \\
\hline 31,0 & - & 18,3 & 16,0 & 375,0 & 26,0 & 175,0 & 14,0 & 31,8 & 35,0 \\
\hline 37,0 & 18,8 & 19,5 & 17,2 & 375,0 & 40,0 & & & & \\
\hline 45,0 & & 20,6 & 18,3 & 200,0 & 32,0 & 175,0 & 8,0 & 46,7 & 20,0 \\
\hline 50,0 & 16,4 & 21,2 & 18,9 & 200,0 & 40,0 & & & & \\
\hline
\end{tabular}

em que: $\mathrm{dg}=$ diâmetro de área basal média, em $\mathrm{cm}$; hdom = altura dominante, em $\mathrm{m}$; hm = altura total média, em $\mathrm{m}$; $\mathrm{N} / \mathrm{ha}=$ número de árvores por hectare; G/ha = área basal por hectare. 


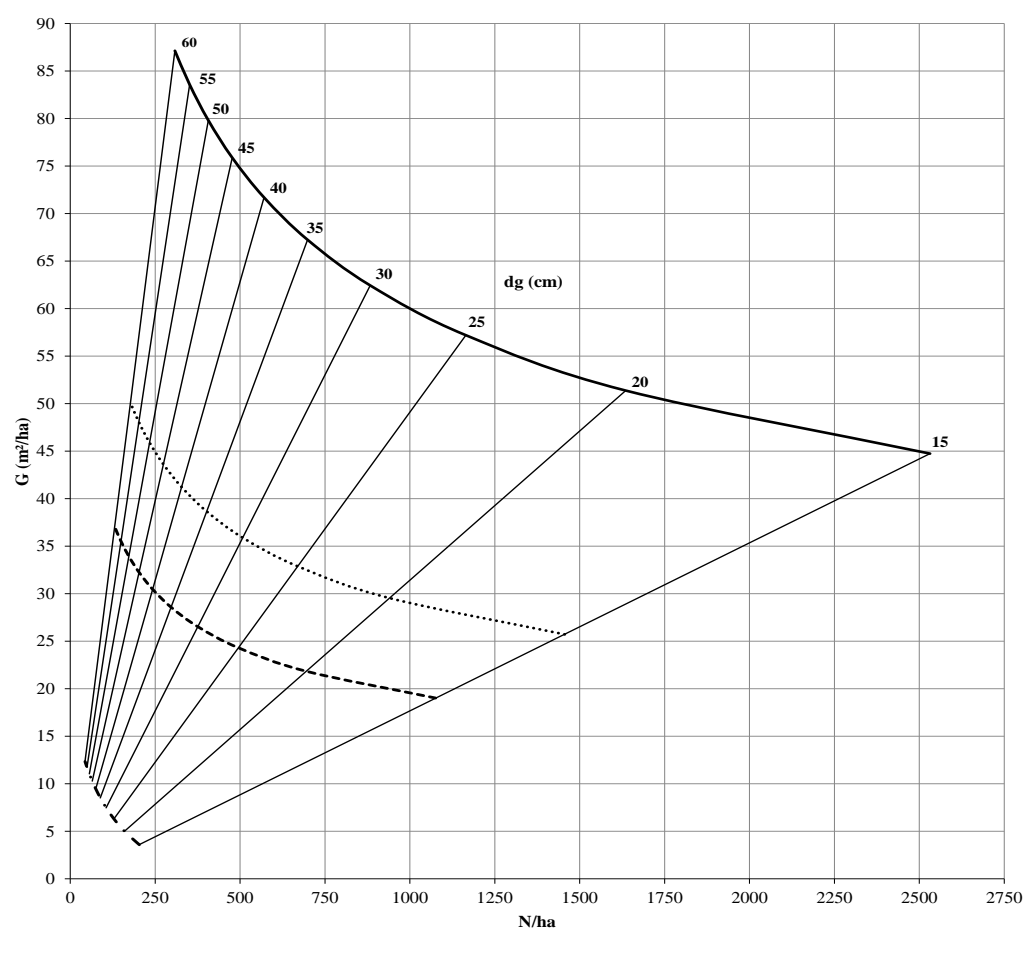

Figura 4. Dendrograma de manejo da área basal (G/ha) relacionado com o número de árvores (N/ha) por hectare e diâmetro de área basal média (dg) para uma Floresta de Araucária, em Lages, SC.

Figure 4. Management dendrogram of basal area $(\mathrm{G} / \mathrm{ha})$ related with the number of trees $(\mathrm{N} / \mathrm{ha})$ per hectare and average basal area diameter (dg) for an Araucaria Forest, in Lages, SC.

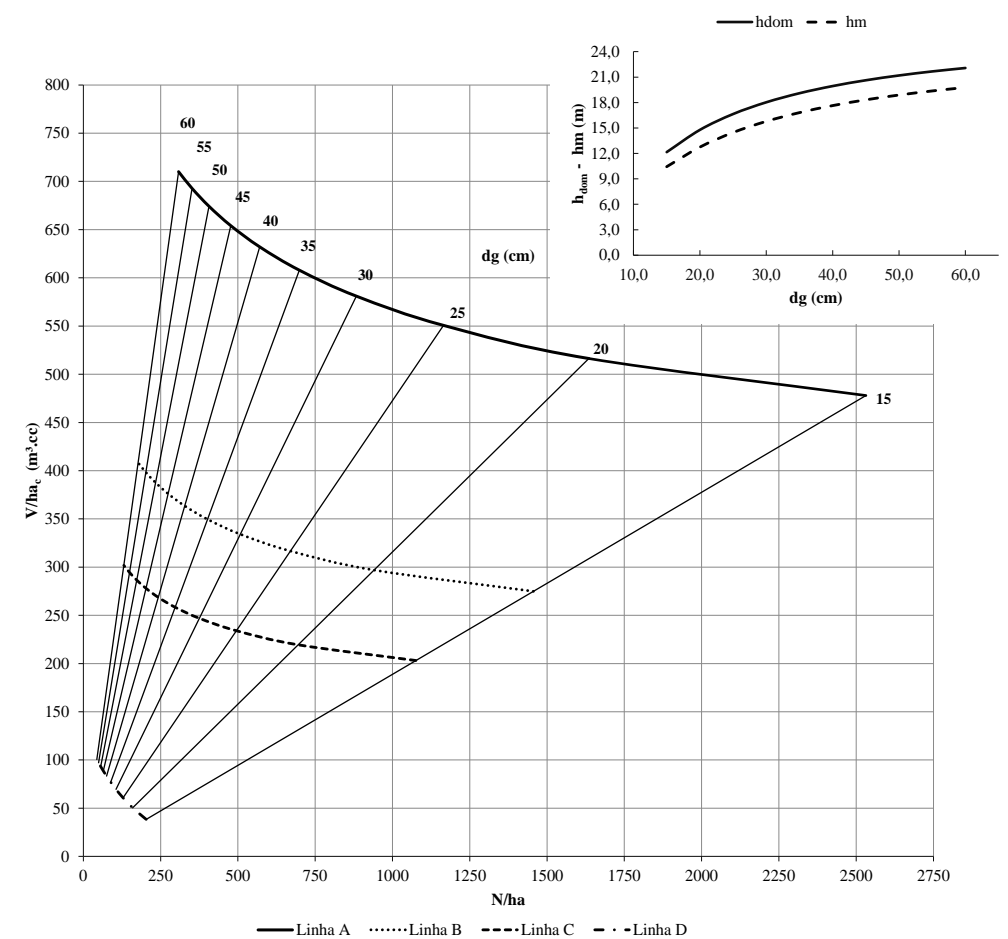

Figura 5. Dendrograma de manejo do volume comercial $\left(\mathrm{V} / \mathrm{ha}_{\mathrm{c}}\right)$ relacionado com o número de árvores $(\mathrm{N} / \mathrm{ha})$ por hectare e diâmetro de área basal média (dg) para uma Floresta de Araucária, em Lages, SC.

Figure 5. Management dendrogram comercial volume $\left(\mathrm{V} / \mathrm{ha} \mathrm{a}_{\mathrm{c}}\right)$ related with the number of trees $(\mathrm{N} / \mathrm{ha})$ per hectare and average basal area diameter (dg) for an Araucaria Forest, in Lages, SC.

FLORESTA, Curitiba, PR, v. 46, n. 2, p. 173 - 184, abr. / jun. 2016.

Costa, E. A. et al.

ISSN eletrônico 1982-4688 
No DMD, foram inseridos os dados observados em campo, esses contemplaram grande parte da amplitude de variação da população em relação as variáveis $\mathrm{G} / \mathrm{ha}$, dg e N/ha (Figura 6). Com a simulação no DMD, evidenciaram-se a necessidade de dois desbastes até obter árvores com as dimensões desejadas, que foi delimitada em um dg de 50,0 cm e G/ha de $40 \mathrm{~m}^{2} . \mathrm{ha}^{-1}$. Com base nos dados de incremento periódico anual em diâmetro obtidos para árvores de araucária no mesmo local do presente estudo (COSTA, 2015), foi possível definir o incremento médio anual em diâmetro de $0,32 \mathrm{~cm} \cdot \mathrm{ano}^{-1}$, entre o dg atual de $31,0 \mathrm{~cm}$ a $37,0 \mathrm{~cm}$, com estimativa de 18,8 anos para a recuperação do estoque (Figura 6).

Deve ser destacado que o incremento médio anual em diâmetro usado para o cálculo da estimativa da idade de recuperação do estoque da floresta foi estabelecido tendo como referência a média dos incrementos de árvores de araucária. Isso pode ser realizado em decorrência da maior dominância da araucária neste ambiente, e como forma de facilitar a exemplificação prática do DMD. Da mesma maneira, foram realizados os demais procedimentos para o segundo desbaste, sendo feito o corte até as dimensões desejadas. Estas intervenções têm como finalidade regular a floresta para que as árvores possam aumentar sua dimensão, que, atualmente, encontra-se com crescimento estagnado, visando, então, alcançar a maximização da produção florestal.

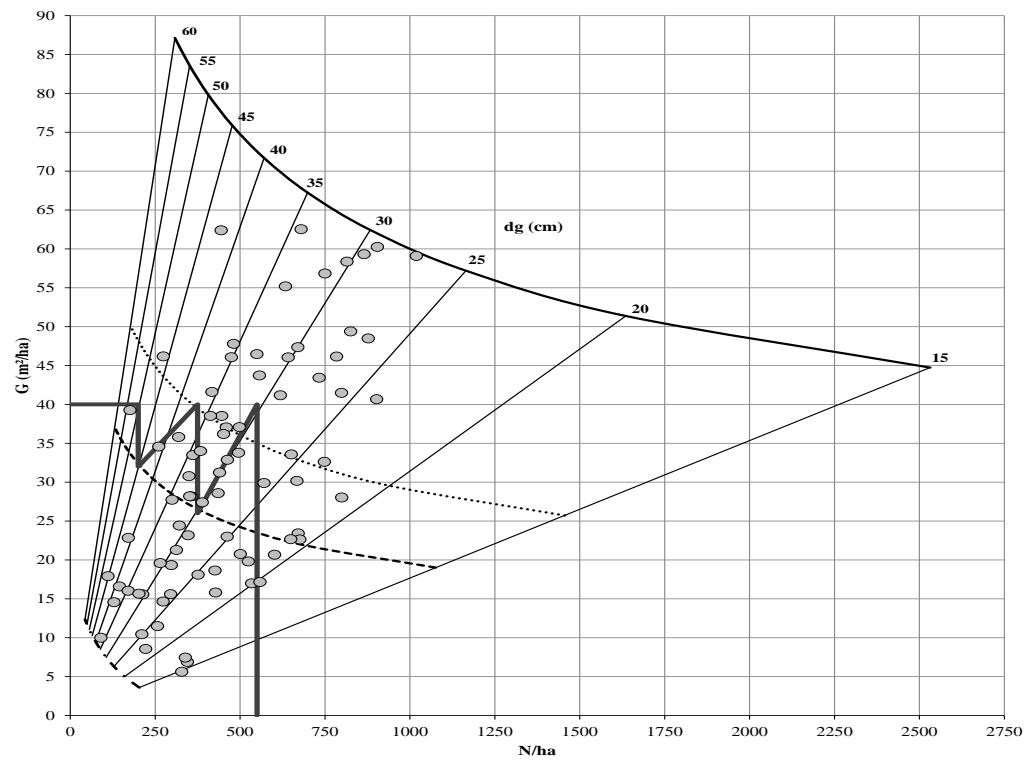

Figura 6. Avaliação e simulação das atividades de desbastes no dendrograma de manejo da densidade da área basal (G/ha) relacionado com o número de árvores por hectare (N/ha) e diâmetro de área basal média (dg), para uma Floresta de Araucária, em Lages, SC.

Figure 6. Evaluation and simulation of thinning activities in the density management dendrogram of basal area $(\mathrm{G} / \mathrm{ha})$ related with number of trees per hectare (N/ha) and average basal area diameter (dg) for Araucaria Forest in Lages, SC.

\section{DISCUSSÃO}

O modelo ajustado de Reineke (1933) é estatisticamente significante, permitindo expressar a tendência média do ln (N/ha) em função do ln (dg) (Figura 2). Embora o $\mathrm{R}^{2}$ tenha sido baixo, o Syx\% teve valor inferior a 10\%, mostrando alta precisão (DRAPER; SMITH, 1998). O valor encontrado para o coeficiente angular do modelo ajustado de Reineke (1933), confirmou a lei da "Potência" -3/2 de "Autodesbaste" formulada por Yoda et al. (1963), que relaciona o tamanho das árvores, neste caso, expresso pelo diâmetro de área basal média (dg) com a densidade populacional (N/ha), evidenciando que o processo de autodesbaste está ocorrendo, tal constante é independente da espécie, localização, idade, densidade inicial e qualidade do sítio (ZEIDE, 1987).

Apesar da grande variabilidade das árvores, os modelos ajustados para o Volume Médio Comercial (VMC), Altura Dominante $\left(\mathrm{h}_{\mathrm{dom}}\right)$ e Altura Média $(\mathrm{Hm})$ (Tabela 3), permitiram descrever essas variáveis de maneira satisfatória, uma vez que, trata-se de florestas naturais com árvores de diferentes formas, dimensões e idades (COSTA et al., 2016).

Estudos vêm sendo desenvolvidos com a finalidade de manejar florestas naturais em ambientes superestocados (HESS et al., 2010; HESS, 2012; HESS et al., 2014), esses autores buscam alcançar o conceito de sustentabilidade da floresta com a regulação da frequência de indivíduos pelo quociente de Liocourt, maximizando o crescimento de árvores individuais e, consequentemente, da produção. 
Beckert et al. (2014) avaliando o crescimento e dinâmica de araucária, encontrou baixas taxas de incremento, devido à intensa competição e à pouca disponibilidade de luz nos estratos inferiores. Os mesmos autores relacionam a esses fatores uma superioridade nas taxas de mortalidade em relação às de ingresso, o que pode indicar uma tendência de desaparecimento da espécie se nenhuma intervenção silvicultural for efetuada.

Para araucária foi verificado o efeito da competição nas relações interdimensionais, na morfometria da copa e no crescimento diamétrico (COSTA, 2015). Esse autor destaca que o controle das árvores a permanecer na floresta durante intervenções de manejo não deve ser baseado apenas na densidade e área basal do local, mas em conjunto com características de cada indivíduo, como o grau de competição entre árvores, posição sociológica, comprimento de copa, entre outros.

Longhi (2011) avaliando o manejo experimental de uma Floresta de Araucária indicou a realização de cortes seletivos leves, redução em torno de $20 \%$ da área basal por classe de DAP, e com ciclos de corte curtos em torno de oito anos, como forma de conduzir a floresta com cortes frequentes a uma estrutura mais produtiva.

Nesse sentido, os DMD's desenvolvidos nesse estudo, visam auxiliar as atividades florestais de silvicultura e manejo florestal para espécie, principalmente em ambientes superestocados, em área de predominância natural, permitindo simular a produção e definir estratégias apropriadas para melhorar o planejamento florestal.

\section{CONCLUSÕES}

- Os modelos ajustados para a confecção do DMD permitem expressar, graficamente, o desenvolvimento das variáveis de controle do crescimento. O coeficiente angular do modelo de Reineke confirma a Lei da Potência de $-3 / 2$ de autodesbaste.

- Os dendrogramas de manejo desenvolvidos para área basal, volume comercial em função do número de árvores por hectare e diâmetro de área basal média nessa floresta de araucária, possibilitaram estabelecer estratégias de manejo florestal, buscando otimizar a produção florestal de acordo com as condições do local e as características de crescimento das espécies, com vistas ao estabelecimento de regime sustentado.

\section{REFERENCIAS}

ALVARES, C. A.; STAPE, J. L.; SENTElHAS, P. C.; GONÇALVES, J. L. M.; SPAROVEK, G. Köppen's climate classification map for Brazil. Meteorologische Zeitschrift, Stuttgart, v. 22, p. 711 - 728, 2013.

BECKERT, S. M.; ROSOT, M. A. D.; ROSOT, N. C. Crescimento e dinâmica de Araucaria angustifolia (Bert.) O. Ktze. em fragmento de Floresta Ombrófila Mista. Scientia Forestalis, Piracicaba, v. 42, n. 102, p. 209 - $218,2014$.

BORSOI, G. A. Subsídios para o manejo de uma Floresta Ombrófila Mista em estágio avançado de regeneração natural. 2004. 163 f. (Doutorado em Engenharia Florestal), Universidade Federal de Santa Maria, Santa Maria. 2004.

CASTEDO-DORADO, F.; CRECENTE-CAMPO, F.; ÁlVAREZ-ÁlVAREZ, P.; BARRIO-ANTA, M. Development of a stand density management diagram for radiata pine stands including assessment of stand stability. Forestry, Oxford, v. 82, p. 1 - 16, 2009.

COSTA, E. A. Modelagem biométrica de árvores com crescimento livre e sob competição em floresta de araucária. 235 f. (Doutorado em Engenharia Florestal), Universidade Federal de Santa Maria, Santa Maria. 2015.

COSTA, E. A.; FINGER, C. A. G.; FLEIG, F. D. Influência da posição social nas relações morfométricas de Araucaria angustifolia. Ciência Florestal, Santa Maria, v. 26, n. 1, p. 225 - 234, 2016.

DEL RÍO, M.; MONTERO, G.; BRAVO, F. Analysis of diameter-density relationships and self-thinning in nonthinned even-aged Scots pine stands. Forest Ecology and management, Amsterdan, v. 142, p. 79 - 87, 2001.

DRAPER, N. R.; SMITH, H. Applied regression analysis. $3^{\circ}$ ed. New York: Wiley, 1998. 706 p.

EMPRESA BRASILEIRA DE PESQUISA AGROPECUÁRIA (EMBRAPA). Sistema brasileiro de classificação de solos. Brasília: Embrapa Produção de Informação; Rio de Janeiro: Embrapa Solos, 2006. 306 p.

HESS, A. F. Manejo de Araucaria angustifolia pelo quociente de Liocourt em propriedade rural no Município de Painel, SC. Pesquisa Florestal Brasileira, v. 32, p. 227 - 232, 2012.

HESS, A. F.; CAlgarotTO, A. R.; PINHEIRO, R.; WANGINIAK, T. C. R. Proposta de manejo de Araucaria angustifolia (Bertol.) Kuntze utilizando o quociente de Liocourt e análise de incremento, em propriedade rural no município de Lages, SC. Pesquisa Florestal Brasileira, v. 30, p. 337 - 345, 2010.

HESS, A. F.; MINATTI, M.; FERRARI, L.; PINTRO, B. A. Manejo de Floresta Ombrófila Mista pelo método de Liocourt, Município de Painel, SC. Cerne, v. 20, p. 575 - 580, 2014. 
KRAJICEK, J. E.; BRINKMAN, K. A.; GRINGRICK, S. F. Crown competition: a measure of density. Forest Science, Bethesda, v. 7, n. 1, p. 35 - 42, 1961.

LONG, J. N.; SHAW, J. D. A. Density Management Diagram for Even-Aged Sierra Nevada Mixed-Conifer Stands. Western Journal of Applied Forestry, Bethesda, v. 27, p. 187 - 195, 2012.

LONGHI, R. V. Manejo experimental de uma Floresta Ombrófila Mista Secundária no Rio Grande do Sul. 2011. 83 f. Dissertação (Mestrado em Engenharia Florestal), Universidade Federal de Santa Maria, Santa Maria. 2011.

LÓPEZ-SÁNCHEZ, C.; RODRÍGUEZ-SOALLEIRO, R. A. Density Management Diagram Including Stand Stability and Crown Fire Risk for Pseudotsuga Menziesii (Mirb.) Franco in Spain. Mountain Research and Development, Bern, v. 29, p. 169 - 176, 2009.

LOUREIRO, G. H. Diagrama de manejo da densidade para Araucaria angustifolia (Bertol) Kuntze. 2013. 72 f. Dissertação (Mestrado em Engenharia Florestal) - Universidade Federal do Paraná, Curitiba, 2013.

MARANGON, G. P. Otimização bioeconômica do regime de manejo para Eucalyptus grandis W. Hill no estado do Rio Grande do Sul. 2015. 196 f. (Doutorado em Engenharia Florestal) - Universidade Federal de Santa Maria, Santa Maria, 2015.

MÁRQUEZ-LINARES, M. A.; ALVAREZ-ZAGOYA, R. Construcción de una guía de densidad para Pinus cooperi var. ornelasi con base en la classe de copa em Durango, México. Madera y Bosques. v. 1, p. 23 - 36.1995.

MEYER, E. A. Densidade de árvores por diâmetro na floresta estacional decidual no Rio do Grande Sul. 2011. 71 f. Dissertação (Mestrado em Engenharia Florestal) - Centro de Ciências Rurais, Universidade Federal de Santa Maria, Santa Maria, 2011.

MICHAILOFF, I. Zahlenmäßiges Verfahren für die Ausführung der Bestandeshöhenkurven. Forstwissenschaftliches Centralblatt und Tharandter Forstliches Jahrbuch, Berlin, v. 6, p. 273 - $279,1943$.

NEWTON, P. F. Stand density management diagrams: Review of their development and utility in stand-level management planning. Forest Ecology and Management, Amsterdam, v. 98, p. 251 - 265, 1997.

PENNER, M.; SWIFT, D. E.; GAGNON, R.; BRISSETTE, J. A stand density management diagram for balsam fir in New Brunswick. The Forestry Chronicle, Mattawa, v. 82, p. 700 - 711, 2006.

REINEKE, L. H. Perfecting a stand-density index for even-aged forests. Journal of Agricultural Research, Lahore, v. 46, p. 627 - 638, 1933.

SAS. The SAS System for Windows. Cary: SAS Institute. 2004.

SCHNEIDER, P. R.; FINGER, C. A. G.; SCHNEIDER, P. S. P.; FLEIG, F. D.; THOMAS, C.; FARIAS, J. A. Quociente do diâmetro pela área basal e zonas de competição em povoamento monoclonal de Eucalyptus saligna. Ciência Florestal, Santa Maria, v. 21, n. 4, p. 755 - 764, 2011.

SCHNEIDER, P. S. P.; FLEIG, F. D.; SCHNEIDER, P. R.; FINGER, C. A. G.; CHAVES, D. M.; MOREIRA, M. M. Diagrama de manejo da densidade para povoamento de Pinus taeda L. conduzido sem desbaste. Ciência Florestal, Santa Maria, v. 19, n. 4, p. 433 - 447, 2009.

SCHUMACHER, F.; HALL, F. Logarithmic expression of timber-tree volume. Journal of Agricultural Research, Lahore, v. 47, p. 719 - 734, 1933.

SELlE, G. L.; FlEIG, F. D.; SCHNEIDER, P. R.; ALBERNARD, L. A. J.; VUADEN, E.; BRAZ, E. M. Dendrogramas de densidade para Hovenia dulcis Thunberg na Região Central do Estado do Rio Grande do Sul, Brasil. Ciência Florestal, Santa Maria, v. 20, n. 3, p. 477 - 492, 2010.

SPURR, S. A measure of point density. Forest Science, Bethesda, v. 8, n. 1, p. 85 - 96, 1962.

THOMAS, C. Dendrograma de manejo da densidade para povoamentos de Pinus elliottii Engelm na região central do Rio Grande do Sul. 2009. 62 f. Dissertação (Mestrado em Engenharia Florestal) - Universidade Federal de Santa Maria, Santa Maria, 2009

VACCHIANO, G.; MOTTA, R.; LONG, J. N.; SHAW, J. D. A density management diagram for Scots pine (Pinus sylvestris L.): A tool for assessing the forest's protective effect. Forest Ecology and Management, Amsterdam, v. 255, p. 2542 - 2554, 2008.

YODA, K.; KIRA, T.; OGAWA, H.; HOZUMI, K. Self-thinning in overcrowed pure stands under cultivated and natural conditions. Journal of biology, Osaka, v. 14, p. 107 - 129, 1963.

ZEIDE, B. Analysis of the 3/2 power-law of self-thinning. Forest Science, Madison, v. 33, p. 517 - 537, 1987. 